\title{
Quelques remarques sur la réécriture chez Montherlant: Mais aimons-nous ceux que nous aimons? et Garder tout en composant tout
}

\section{Claude Coste}

\section{(2) OpenEdition}

Journals

Édition électronique

URL : https://journals.openedition.org/genesis/5756

DOI : 10.4000/genesis.5756

ISSN : 2268-1590

Éditeur :

Presses universitaires de Paris Sorbonne (PUPS), Société internationale de génétique artistique littéraire et scientifique (SIGALES)

\section{Édition imprimée}

Date de publication : 15 décembre 2020

Pagination : 213-221

ISBN : 979-10-231-0704-3

ISSN : $1167-5101$

\section{Référence électronique}

Claude Coste, "Quelques remarques sur la réécriture chez Montherlant : Mais aimons-nous ceux que nous aimons ? et Garder tout en composant tout », Genesis [En ligne], 51 | 2020, mis en ligne le 20 décembre 2021, consulté le 07 février 2022. URL : http://journals.openedition.org/genesis/5756 ; DOI : https://doi.org/10.4000/genesis.5756 


\title{
Quelques remarques sur la réécriture chez Montherlant : Mais aimons-nous ceux que nous aimons? et Garder tout en composant tout
}

\author{
Claude Coste
}

$\mathrm{M}$ ontherlant a réécrit toute sa vie. Marquée du sceau de l'ambiguïté - «toute sa vie» est-il complément de temps ou d'objet? -, la réécriture est, pour l'auteur du Songe et de La Ville dont le prince est un enfant, une pratique essentielle qui a été analysée de façon ponctuelle par les chercheurs. Un tel sujet attend toujours sa thèse ou son livre d'envergure pour mesurer l'importance du phénomène dans la totalité de l'œuvre - rappelant combien les acquis de la critique génétique sont à même d'enrichir le renouveau des études montherlantiennes. D'une ambition beaucoup plus modeste, cet article se contentera de formuler quelques «remarques sur la réécriture», et d'apporter une simple pierre à l'édifice, à partir d'un corpus restreint et cohérent. Deux ouvrages posthumes, de nature et de statut très différents, ont paru, l'un en 1973, Mais aimons-nous ceux que nous aimons ?', l'autre en 2001, Garder tout en composant tout ${ }^{2}$, qui font, tous les deux, de la réécriture un enjeu fondamental de la création littéraire.

Mais aimons-nous ceux que nous aimons ?, livre difficilement classable, à la fois essai et roman, ne semble pas très bien savoir où il va. Très volontairement, l'auteur court plusieurs lièvres à la fois : le livre naît d'une réaction agacée à la relecture du Songe, paru en 1922, roman de jeunesse qui raconte les amours chastes d'Alban de Bricoule et de Dominique, une jeune fille qu'il rencontre sur un stade. C'est cette chasteté un peu mystérieuse, liée au monde du sport, que le vieil écrivain tente d'expliciter dans son ouvrage quasi testamentaire. De ce premier objet, Montherlant passe à une réflexion plus vaste sur la réalité de l'amour, qui lui permet, en suivant un cheminement très rapsodique, d'évoquer de nombreux épisodes de son enfance ou de sa vie d'adulte, les uns très drôles, les autres plus tragiques quand il s'agit de la guerre et de la mort, omniprésente dans tout le livre. La conclusion de la quête et de l'enquête impose un bilan dominé par le noir, à peine teinté de gris :

\begin{abstract}
L'Hamour, lui, cette inexistence passionnée, n'en parlons même pas. [...] Nous n'aimons que des moments, et toutefois, en ayant conscience, c'est une conscience que nous devons surmonter, car il faut aimer. Il faut vivre dans cette illusion et dans cette clairvoyance : elles sont l'une et l'autre l'honneur de l'homme, et les juxtaposer est encore à son honneur. (210-211).
\end{abstract}

Se produit alors un magnifique coup de théâtre : dans les toutes dernières pages du livre, Montherlant est submergé par l'intrusion d'un rêve bouleversant, qui lui apprend qu'il a aimé au moins une fois dans sa vie. Or le récit de ce rêve poignant, pour l'auteur comme pour son lecteur, est le fruit d'une réécriture très minutieuse dont Garder tout en composant tout permet de mesurer le cheminement.

Gardons tout en composant tout ne présente pas l'unité d'Aimons-nous. Il s'agit, sous la direction de Yasmina et de Jean-Claude Barat, d'un recueil composite de textes, parfois très courts, demeurés à l'état de manuscrit dans les archives de Montherlant. On y trouve des fragments inédits, des brouillons d'articles publiés dans plusieurs organes de presse, de nombreux passages qui seront réécrits par la suite pour être incorporés dans telle ou telle œuvre. En rapprochant ces deux ouvrages posthumes, si différents de nature, le lecteur se trouve confronté à une pratique de dialogisme avec soi-même dont les manifestations présentent une grande diversité. Dans Aimons-nous..., Montherlant met en scène sa relecture d'un texte de jeunesse (Le Songe), puis propose une nouvelle version de la Petite 19, nouvelle

\footnotetext{
1. Le titre est emprunté à Maurice Clavel, Paris, Gallimard, coll. «nrf», 1973. Les références de pages figureront entre parenthèses après la citation.

2. Garder tout en composant tout (1924-1972), Carnets inédits - Derniers carnets, textes réunis, annotés et présentés par Jean-Claude et Yasmina Barat, Paris, Gallimard, coll. «les Cahiers de la NRF», 2001. Les références de pages seront données entre parenthèses, après la citation.
} 
écrite, elle aussi, au cours des années vingt et racontant une histoire sentimentale, cette fois-ci beaucoup moins chaste, dans le monde du sport. La réécriture est clairement mise en avant par Montherlant, qui revient à la fin de sa vie sur des écrits de jeunesse qui l'agacent ou ne le satisfont plus. Le regard rétrospectif et le travail de correction s'installent presque théâtralement au cœur de la création. Le cas de Garder tout... est totalement différent. Grâce à l'exécuteur testamentaire, le lecteur découvre des brouillons, livrés un peu pêle-mêle, qui lui donnent l'occasion de lire une première version du rêve publié dans Aimons-nous... Cette fois-ci, ce n'est plus l'auteur qui met en scène sa pratique de la réécriture, c'est le lecteur de Garder tout... qui reconstitue le cheminement créateur d'une version à l'autre - l'une publiée par l'auteur, la seconde par son héritier. Il n'empêche : au-delà des différences entre une réécriture exhibée et une réécriture restée au secret dans les archives, il s'agit toujours pour Montherlant de revenir sur son travail et de corriger le passé. Par exemple, lors de l'édition des Essais dans la Pléiade en 1963, et en particulier du si controversé Solstice de juin, l'auteur tout puissant a bien pris soin de couper ou modifier les passages compromettants sur son attitude pendant l'Occupation. Mais les préoccupations politiques sont loin d'expliquer, à elles seules, cette passion de la reprise, de la redite, de la reformulation qui habite toute son œuvre. En réécrivant sans cesse ses textes, Montherlant entend rester maître du jeu; et pour ceci façonne perpétuellement son masque ou son visage, afin de dessiner le portrait qu'il veut laisser de lui-même - et principalement dans des domaines aussi sensibles que la vie sentimentale et le désir sexuel. Pour un homme qui voit dans chaque sensation une source de création et une garantie de sincérité, la réécriture, en remettant le code et les mots au cœur du travail littéraire, nous interroge sur la mystérieuse cohabitation de l'émotion et de la ruse.

\section{Nuages}

Par où commencer? La réponse surprendra peut-être : par les nuages. Ou plutôt par la métaphore des nuages qui est peut-être la métaphore de toutes les métaphores dans l'œuvre de Montherlant. Cette image apparaît dans les Carnets, dans Le Cardinal d'Espagne ${ }^{3}$, dans les deux ouvrages posthumes ${ }^{4}$. Et Montherlant de manifester une claire conscience des traits récurrents de son écriture, comme il le précise dans Garder tout... :

Je remarque une fois de plus que dans mes œuvres deux comparaisons sont plus que des comparaisons, sont des images qui collent minutieusement sur la réalité, sont des images-réalités :

la marée du soir et les nuages de la reine Jeanne. (410)

Associée à la reine Jeanne, plus sage dans sa folie que le politique cardinal Cisneros, la métaphore dit beaucoup sur le spectacle du monde tel que le perçoit et le décrit Montherlant. Dans cette formule, on retrouve le syncrétisme et l'alternance, le pessimisme et le désenchantement, une appréhension presque baroque d'un univers dominé par l'inconstance, tantôt noire, tantôt blanche (les «merveilleux nuages » dont parlait Baudelaire).

Mais dans sa diversité, la métaphore des nuages renvoie également à la réécriture elle-même, quand elle conjugue deux réalités apparemment contradictoires : la permanence et la transformation. En se métamorphosant sans cesser d'être lui-même, le nuage entre en relation avec le travail de l'écrivain qui modifie sa phrase ou son texte, impose des corrections de détail, infléchit le sens, sans pour autant modifier la signification générale de l'œuvre. De livre en livre, mais aussi d'état en état du texte, la philosophie et la poétique de Montherlant accommodent la permanence d'une vision du monde et le dynamisme d'un univers en perpétuel changement. C'est incontestablement une constante volonté de maîtrise qui assure l'unité entre ce qui demeure et ce qui se transforme. On peut même aller plus loin : face à un monde qui change en permanence, face à une écriture qui engendre souvent la réécriture, Montherlant s'impose comme le régisseur de ses propres

3. «Il n’y avait pas de nuages. Maintenant, il y en a. Ils vont changer d'aspect. Ils vont se dissiper, puis se reconstituer d'autre façon. Tout cela est sans importance. Des nuages noirs étalés sur Madrid comme de gros crapauds », Le Cardinal d'Espagne, dans Théâtre, préface de Jacques Laprade, préface complémentaire de Philippe de Saint-Robert, Paris, Gallimard, coll. «Bibliothèque de la Pléiade», 1972, p. 1145.

4. On peut lire dans Garder tout... : «Celui qui lit ces lignes et qui est indigné s'apercevra un jour qu'elles sont vraies. Ou plutôt il ne s'en apercevra pas, car il les aura oubliées, oubliées comme il avait oublié les objets de sa tendresse. Dissous, comme les nuages de la reine Jeanne.» (385) 
nuages. Relire et réécrire un roman de jeunesse comme Le Songe, revoir toute son œuvre ancienne quand il s'agit de la monumentaliser dans la Pléiade, travailler ses brouillons pour en cacher ou pour en exhiber les métamorphoses : la volonté de Montherlant s'impose aux mots et aux lecteurs.

Le travail de réécriture porte sur l'énoncé, comme on s'y attend. Dans l'ensemble, il s'agit moins d'infléchir l'idée générale que de modifier le détail de l'expression - ce qui a bien sûr pour effet d'altérer, même légèrement, cette signification générale. On connaît le goût de Montherlant pour le «style», qu'il faut comprendre en relation avec sa formation classique, tout imprégnée de culture antique et de XVII siècle. Dans ce sens-là, réécrire, c'est mieux écrire, c'est faire plus dense, c'est s'inspirer de la phrase latine, plus synthétique, et d'un art de la formule qui trouve dans la maxime une de ses réalisations les plus prestigieuses. Ainsi la poétique de la réécriture passe souvent par une pratique de la coupure et de la réduction. Ce qui vaut pour le théâtre - un art qui n'a pas toujours le temps de prendre son temps - vaut pour tous les genres traités par l'auteur : «Moi-même, plus tard, j'ai vu que l'essentiel n'est pas de faire des pièces, ni même de faire des dédicaces, mais de faire des coupures », écrit-il dans Garder tout... (182). En condensant la matière verbale, Montherlant participe à un imaginaire de la littérature qui associe étroitement la réduction, la densité et la vérité. Hanté par la maxime, il travaille à faire tenir la plus grande généralité de sens dans une forme aussi ramassée que possible, comme si la vérité se gagnait pas à pas contre l'abondance des mots. Voici la première version d'une anecdote, parue dans Garder tout... :

Ô homme admirable, qui venait m'entretenir de je ne sais quoi de littérature, à qui j'avais réservé trois quarts d'heure de ma vie et qui n'en resta qu'un! Une visite de quinze minutes, ô homme sublime ! homme exemplaire! Ô homme-des-quinzeminutes, archétype des visiteurs de toute espèce et saint pour les visités! (151)

Et voici la version publiée dans le Carnet XXI : «Un inconnu devait venir chez moi, pour affaires. J'avais calculé qu'il resterait une heure. Il reste quinze minutes. Enthousiasmé, je m'en fis un ami5.»

Mais, liée à un imaginaire de la réduction commun à tant d'écrivains contemporains, la réécriture ne se limite pas à un art de l'économie. La Petite 19, cette nouvelle de 1923, n'est pas seulement reproduite, mais aussi largement modifiée dans Mais aimons-nous... De toutes les raisons avancées par l'auteur, on note incontestablement la volonté de réduire le lyrisme un peu démodé et bavard du héros, de changer tel ou tel mot qui ne parle plus beaucoup aux Français de 1973 («weatherproof» est remplacé par «imperméable»). Mais les corrections stylistiques importent moins que la volonté d'éclairer le lecteur. Or, «expliquer», c'est étymologiquement «développer». En relisant Le Songe, Montherlant s'explique longuement sur la chasteté des deux personnages principaux, la mettant entièrement sur le compte du sport et ses exigences. Avec La Petite 19, en passant de la relecture à la réécriture, le projet reste le même. Il s'agit là encore de tenir à distance le monde masculin et de lever le soupçon sur l'homosexualité latente du texte. La confrontation de nombreux passages montre avec évidence que la présence de la femme, «la petite 19 », est bien plus nettement affirmée dans la seconde version comme s'il s'agissait d'écarter les mauvaises interprétations 6 .

C'est aussi dans cette étrange Petite 19 que l'on retrouve la fameuse métaphore des nuages, mais selon un emploi qui en dit long sur la diversité des projets de Montherlant. Le héros est sur le point de formuler une remarque peu aimable sur les femmes; un reste d'humanité et de galanterie l'amène à corriger ses pensées :

J'allais dire : «Ton âme m'est indifférente. Il n'est même pas nécessaire que tu aies une âme.» Nuageons cela un peu. Faisons-en un alexandrin, de façon que ça ne tire plus à conséquence :

Je crois que j'ai besoin plus des corps que des âmes.

Et c'est un allègement de tout mal. (123)

5. Henry de Montherlant, Essais, préface de Pierre Sipriot, Paris, Gallimard, coll. «Bibliothèque de la Pléiade», 1963, p. 1055.

6 . On se contentera d'un exemple : «Je te dirai un jour comment, sur le point d'avoir le mal de mer, ou, sous le soleil torride, sentant l'étourdissement venir, j'ai dompté la menace en me représentant avec force ton visage, ton attache du cou», lit-on dans Aimons-nous... (123). À comparer avec la version originale de 1923 : «Cette forme pure [1'alexandrin] me pénètre comme la bonne huile que presse en moi le masseur. Je te dirai un jour comment, sur le point d'avoir le mal de mer, ou, sous le soleil torride, sentant l'étourdissement venir, j'ai dompté la menace en me représentant avec force tel visage, telle attache du cou, moins encore, un des fantômes de l'art, la figurine tracée sur un vase grec.» 
S'ils font leur retour, les nuages du jeune mufle ne ressemblent pas vraiment aux nuages de la reine Jeanne. Que signifie exactement le verbe «nuager»? Montherlant joue manifestement sur la polysémie des connotations : «nuager» implique une fois encore la métamorphose puisqu'il s'agit de transformer ou d'édulcorer une mauvaise pensée. Mais si l'on se réfère au dictionnaire, «nuager», c'est d'abord «couvrir de nuages», c'est-à-dire cacher le ciel et voiler ses pensées. Quant à l' ' allègement » obtenu, il n'est pas étranger à l'immatérialité du nuage qui semble aussi léger que l'air. De la reine Jeanne au jeune sportif, les «nuages » ont changé moins de forme que de fonction; à la simple transformation, ils ajoutent les pouvoirs de la dissimulation et de l'aération?.

À ce premier travail sur l'énoncé, correspond un autre type de réécriture qui porte cette fois-ci sur l'énonciation elle-même. En d'autres termes, il ne s'agit plus de modifier le contenu, mais de le déplacer vers un autre émetteur, vers un autre sujet. Les fonctions de ce nomadisme de la parole sont multiples. On relèvera en particulier la volonté de lutter contre les dangers de la réduction. L'imaginaire de la maxime et son arme, la coupure, s'accompagnent très souvent d'un goût pour l'assertion, pour le discours de savoir qui impose au lecteur des vérités destinées à le surprendre, le choquer ou l'ébranler. Mais toute écriture de moraliste se heurte très souvent au scepticisme qui caractérise les nuages de la reine Jeanne. Travaillé par un imaginaire de la condensation, Montherlant transcende chaque expérience singulière - à commencer par la sienne en expérience collective, pour ne pas dire anthropologique. Mais ce mouvement de métamorphose du singulier vers l'universel risque de sombrer dans l'ubris. Une phrase de Garder tout..., reprise dans les Carnets, se montre sans illusion sur la facticité du théâtre intellectuel : «Moraliste, j'émets des lois générales. / Mais toute loi générale est fausse» (Carnets, 1327). Vertige sans fin d'une affirmation gnomique qui stigmatise la généralité...

Construit sur une pratique de la réduction progressive, le désir esthétique et existentiel de dire moins pour dire au plus près des lois générales de la condition humaine risque à tout moment de virer à la bêtise - et la bêtise du contenu menace de révéler la bêtise de l'énonciateur. Si Balzac n'est jamais bête dans ses descriptions, il l'est parfois comme tant d'écrivains dans ses jugements aphoristiques, ses affirmations à l'emporte-pièce qui s'illusionnent sur le pouvoir de mettre le savoir en forme comme on met le vin en bouteille. Bien sûr, la bêtise n'est jamais sûre; telle phrase (elles ne manquent pas chez Montherlant) qui agacera l'un par son arrogance péremptoire enchantera l'autre par sa justesse de vue (elle ne manque pas chez Montherlant). Mais La Rochefoucauld risque à tout moment de se transformer en Bouvard et Pécuchet ou en M. Perrichon. Quand on écrit des sentences, on finit par devenir sentencieux... C'est le génie de Montherlant de ne jamais demeurer longtemps dupe de lui-même. Une lucidité souvent cruelle explique la prolifération dans son œuvre des ratés, des épaves, des déchets comme MM. de Coantré et de Coëtquidan, les deux célibataires des Célibataires, l'Exupère d'Un assassin est mon maître, et même le pathétique Celestino du Chaos et la Nuit. Chez Montherlant, l'hidalgo n'est jamais bien loin du pauvre type et toute son œuvre est habitée par la paradoxale lucidité de Matamore.

À la puissance, somme toute appauvrissante de la maxime, Montherlant oppose une fois encore l'exemple des nuages. Pour contrer la réduction, il faut compter sur la pluralisation de soi que permettent la littérature en général et le nomadisme des voix en particulier : «Je suis fou à volonté, me mets en colère à volonté, etc., enfin je peux être n'importe quoi, et instantanément : c'est ce qui m'a permis d'écrire les pièces que j'ai écrites, et les romans que j'ai écrits» (182). Garder tout... offre plusieurs exemples de ces énoncés qui changent de porte-parole au gré des réécritures. On retiendra parmi tant d'autres une formule proche de la maxime et de la plus haute généralité : «Quand l'oiseau de race est pris, il ne se débat plus. (proverbe arabe)» (113). Sous sa forme manuscrite, publiée de manière posthume, l'affirmation conjugue déjà deux énonciations : la collectivité que représente la culture arabe, et la singularité de Montherlant qui, d'une certaine manière, reprend l'affirmation à son compte en se l'appropriant. Puis, en demeurant identique à la lettre, la phrase migre dans une pièce de théâtre, La Reine morte, et passe de la généralité culturelle - le monde arabe n'est plus mentionné - et de la singularité d'un auteur à la singularité d'un personnage de

\footnotetext{
7. La critique a montré combien Montherlant réécrit à son avantage de nombreux passages de livres aussi problématiques sur le plan politique que L'Équinoxe de septembre et surtout Le Solstice de juin.
} 
fiction, une femme enceinte et amoureuse ${ }^{8}$. Prononcée par Inès de Castro, la citation devient réplique, le proverbemaxime devient sentence; Montherlant laisse la parole à une jeune femme qui, en apparence au moins, se tient aussi loin de lui que possible. En migrant d'une bouche à l'autre, le savoir perd non pas de sa force, mais de son arrogance; il devient une expérience partageable par tous et adaptable par chacun, il se trouve relativisé par la situation et en même temps investi du poids de la réalité vécue. Cette volonté d'échapper à soi-même pour continuer à être soi, de lutter contre l'assertion de vérités illusoires explique en partie au moins le choix du théâtre après les imprudences commises pendant la guerre par l'usage d'une forme aussi directe que l'essai. Au théâtre, c'est d'abord le personnage qui exprime sa pensée, sans le contrôle d'un narrateur de roman. Au théâtre encore, c'est le comédien qui prend physiquement la parole et impose l'altérité de son timbre, de son débit et de son jeu.

Face à cette dépossession, à cette «nuagisation » de soi, à cette transhumance des maximes, l'attitude de Montherlant est double. D'un côté, il ne fait jamais que ce qu'il veut puisque la fictionnalisation et la dramatisation ne dépendent que de lui-même; d'un autre, il craint cette dépersonnalisation qui est son œuvre. On trouve dans Garder tout... une page beaucoup plus violente que la version publiée, dans lesquelles il avoue le vertige que lui procure l'expérience de la scène :

Les brames du roi Ferrante : «Ah! la mort qui vous met enfin hors d'atteinte.» - «Nous sommes bien loin ici du Royaume de Dieu.» - «Cela est étrange mais il n'y a que des choses étranges par le monde.» Ces cris sortis de ma vie privée, $\mathrm{y}$ rentrèrent avec un accent nouveau, celui qu'y met Yonnel, et à l'heure de ma mort, ils y seront avec cet accent-là. Alliage bizarre de votre moi le plus intime et de ce qui vous est étranger ${ }^{9}$. (135)

Comble de ce vertige que crée le changement d'énonciation, le théâtre présente paradoxalement une autre forme de danger, que Montherlant ne mentionne pas. Toute réplique appartient d'abord au personnage; mais tous les dramaturges connaissent bien les pièges ou les séductions que présentent les «mots d'auteur», ces formules qui par-dessus la tête des personnages expriment directement, pour rire ou pour penser, la voix de l'auteur. Peut-être Montherlant s'est-il cru trop facilement protégé par la double énonciation et par la migration des énoncés. L'accusation a souvent été formulée à son encontre : derrière les protagonistes de son théâtre, on entend trop souvent et trop directement la voix pas assez «nuagée» de l'auteur, un peu comme on entendrait trop Sacha Guitry, Jean Anouilh ou Thomas Bernhardt derrière les fantoches qu'ils exhibent sur la scène. Sans établir de hiérarchies qui resteraient forcément subjectives, c'est peut-être avec le roman que Montherlant réussit le mieux à se métamorphoser sans cesser d'être lui-même.

\section{Un rêve}

Après la généralité des «nuages » et pour mieux comprendre cette pratique de la réécriture qui anime la poétique de Montherlant, il est temps de passer au rêve du 12 décembre 1971 publié dans Mais aimons-nous... et de le confronter à la première version, publiée dans Garder tout..., en 2001 (voir l'encadré de la page suivante, les variants sont grisés).

Grâce à cette double parution, le lecteur dispose de deux versions assez différentes du même rêve. D'un côté, un récit qui constitue un tout; de l'autre, une page qui s'inscrit dans l'économie générale d'un livre. Ce qui frappe d'abord, dans Aimons-nous..., c'est l'effort pour intégrer le rêve dans la problématique générale de l'œuvre, c'est-à-dire à la fois une réflexion générale sur l'amour et une évocation des grandes épreuves formatrices de la jeunesse de Montherlant, comme la guerre ou le sport. Cette volonté à la fois d'arrimage et de mise en écho se donne à voir dès la première phrase qui assure la transition ( Quant à l'amour, c'est par des rêves que je me souviens...») et surtout dans le long passage

8. La Reine morte, op. cit., p. 135.

9. Et voici la version un peu édulcorée de la notice de La Reine morte dans l'édition de la Pléiade (p. 188) : " "Ah! la mort qui vous met enfin hors d'atteinte." - "Nous sommes bien loin ici du Royaume de Dieu." - "Cela est étrange mais il n'y a que des choses étranges par le monde". Ces phrases, sorties de ma vie privée, y rentrent avec un accent nouveau, celui qu' y met Yonnel, et, à l'heure de ma mort, je les y trouverai avec cet accent-là. Alliage bizarre de votre moi le plus intime et d'une personnalité autre que la vôtre.» 


\section{Mais aimons-nous ceux que nous aimons...? \\ (Gallimard, 1973)}

Quant à l'amour, c'est par des rêves que je me souviens et que je sais que je l'ai éprouvé. Je recopie ici la note que j'écrivis le matin du 12 décembre 1971, une heure à peine après mon réveil :

Il y a des rêves prémonitoires. Il y a aussi des rêves révélateurs : qui vous révèlent quelque chose d'important sur vous-même ou sur le monde.

Curieusement, je ne rêve jamais. Pourtant il a fallu qu'en cette fin d'année - les fins d'années toujours sinistres pour moi - me vint un rêve, sans doute très court, pas plus qu'une image; et au petit matin, à l'heure où les rêves empiètent sur la réalité. Dans ce rêve j'ai vu apparaître quelqu'un que j'ai aimé en des temps très lointains. À l'instant j'ai été éveillé par les battements de cour précipités que me donnait l'apparition de ce visage béni. Je me suis tourné sur le côté gauche vers le mur, comme pour étouffer ces battements par le matelas, et il me sembla que je les envoyais à travers le matelas jusqu'au centre de la terre. S'ils m'avaient emporté, si ç'avait été une crise au cœur, quelle fin de vie! À la lettre, mourir d'amour.

Or, ce rêve, je le fais périodiquement. Tous les combien? Mettons tous les six ou sept ans, depuis un temps infini, et chaque fois la matinée du réveil est bouleversée, comme l'a été celle du 12 décembre (mais les battements de cour étaient une nouveauté). Car le rêve du 12 décembre m'a montré que cet être était le seul que j'aie aimé de ma vie entière, que mes autres amours n'avaient été que des caricatures de celui-là, et que le bonheur même avait été peu de chose après lui. Je n'avais pas pensé à cette personne de toute la veille, bien que je pense à elle très souvent. De quel abîme le rêve l'avait-il tirée, pour m'empoisonner d'avance ces derniers jours de l'année, en me découvrant que tout ce qui n'était pas elle n'avait été rien?

Cette visitation silencieuse est bouleversante pour moi parce qu'elle bouleverse toute l'idée que j'avais de ma vie. Moi qui ai répété tant de fois : "Je ne sais qu'aimer», n'aurais-je aimé qu'une fois? Je ne peux pas dire que j'ai cru aimer bien d'autres fois. Non, de vrai, je ne le croyais pas. Il m'est arrivé quand même d'en avoir une certaine illusion, et les mots «d'amour» que je prononçais étaient prononcés de franc cœur; mais non, on n'aime qu'une fois, et cette pensée s'ouvre pour moi sur la désolation.

J'ai lu, et peut-être pensé, et peut-être écrit que tous les êtres sont remplaçables. J'ai su cette nuit, ce petit matin, que cela n'est pas.

[...] L'incommunicabilité d'être à être a existé et a été dénoncée de tous temps. Mais pas au point où elle existe aujourd'hui. Ce rêve clôt un monde fini. Non seulement fini pour moi, parce que je vais finir, mais fini en soi. Dans la société nouvelle qui pourra comprendre encore une certaine qualité de sensibilité, dont toute mon œuvre, depuis mon premier livre, est imprégnée et comme poreuse? Le monde de demain n'en aura

\section{Garder tout en composant tout}

\section{(Gallimard, 2001)}

«On n'aime qu'une fois.»

(Rêve du 12 décembre 1971 au petit matin.)

Il y a des rêves prémonitoires. Il y a aussi des rêves révélateurs : qui vous révèlent quelque chose d'important sur vous-même ou sur le monde.

Je ne rêve jamais. Pourtant il a fallu qu'en cette fin d'année - les fins d'années toujours sinistres pour moi - me vint un rêve, en apparence très court, et au petit matin, à l'heure où les rêves empiètent sur la réalité. Je me suis éveillé à sept heures et, pour autant qu'on puisse préciser ces choses, me suis éveillé au cours du rêve. Dans ce rêve j'ai vu apparaître quelqu'un que j'ai aimé en des temps très lointains et tel qu'il était alors. Il y avait aussi un tiers invraisemblable, d'ailleurs de peu d'importance, et dont je me demande ce qu'il venait faire là.

Ce rêve m'a bouleversé. Il m'a montré le seul être* que j'aie aimé de toute ma vie, et surtout il m'a montré qu'il était le seul, malgré toutes mes illusions sincères; que les autres n'en avaient été que des caricatures; et que le bonheur même avait été peu de chose après lui. Je n'avais pas pensé à cet être de toute la veille, bien que je pense à lui très souvent. De quel abîme le rêve l'avait-elle fait remonter, pour m'empoisonner d'avance ces derniers jours de l'année, les empoisonner en me montrant que tout ce qui n'était pas cet être n'avait été rien?

Ce rêve est bouleversant pour moi parce qu'il bouleverse toute l'idée que j'avais de ma vie. Moi qui ai répété tant de fois : «Je ne sais qu'aimer», n'aurais-je aimé qu'une fois? Je ne peux pas dire que j' ai cru aimer bien d'autres fois. Non, de vrai, je ne le croyais pas. J'en avais quand même une certaine illusion et les mots «d'amour» que je prononçais étaient prononcés de bon cœur; mais non, on n'aime qu'une fois, et cette pensée s'ouvre pour moi sur la désolation.

J'ai lu, et peut-être pensé, et peut-être écrit que tous les êtres sont remplaçables. J'ai su cette nuit, ce petit matin, que cela n'est pas.

J'écris ceci une heure à peine après mon réveil. J'ai voulu noter tout de suite l'impression que m'avait faite ce rêve. Mais déjà le dessin de celui-ci s'efface. Puisse-t-il s'effacer tout à fait.

L'incommunicabilité de l'être à l'être a existé et a été dénoncée de tous temps. Mais pas au point d'aujourd'hui. Ce rêve clôt un monde fini. Non seulement fini pour moi, parce que je vais finir, mais fini en soi. Qui pourra comprendre encore une certaine qualité de sensibilité, dont toute mon œuvre, depuis mon premier livre, est imprégnée et comme poreuse ? Le monde de demain n'en aura pas la moindre notion. Dès aujourd'hui, qui peut comprendre, même si je savais l'exprimer mieux, ce que ce rêve a été pour moi ? Un tel problème ne m'avait jamais arrêté. Depuis ce matin oui. Ce n'est pas le «Ouvrez-vous, portes éternelles» que j'écrivais hier. C'est : «Fermez-vous, portes éternelles ». 
pas la moindre notion. Dès aujourd'hui, qui pourraient comprendre, même si je savais l'exprimer mieux, ce que ce rêve a été pour moi. Un tel problème ne m'avait jamais arrêté. Depuis ce matin, ce n'est pas le «Ouvrez-vous, portes éternelles» que j'écrivais dans un de mes Carnets. C'est «Fermez-vous, portes éternelles».

Si j'ai reproduit ici ma note du 12 décembre 1971, c'est pour corriger l'impression que pourrait donner la conclusion de ce récit. Je n'ai peut-être aimé qu'une fois. Mais, une fois, c'est déjà beaucoup.

Les prétoriens cernaient les bosquets sur les berges de l'étant d'Agrippa.

Juillet-août 1972.
Écrit le soir. - Comme prévu, dès ce soir le rêve s'est effacé de ma mémoire et de ma sensibilité. Ce matin, c'était immense, et ce soir ce n'est rien. Le rêve a existé, mais n'était rien. L'impression qu'il m'a faite a existé, puisque que je l'ai notée sur-le-champ, mais elle n'était rien, puisque née de quelque chose qui n'était rien. Ce sont les nuages de Jeanne la Folle, qui existent, puis changent de forme, puis cessent d'exister.

(Je remarque une fois de plus que dans mes œuvres deux comparaisons sont plus que des comparaisons, sont des images qui collent minutieusement sur la réalité, sont des imagesréalités : la marée du soir et les nuages de la reine Jeanne.)

Écrit trois jours plus tard. - Et pourtant cela a existé. En fait et dans l'impression que j'en ai reçue. En vérité, je ne sais que penser...

(*) Comment nommer ce «quelqu'un»? «La personne»? «la créature»? «L'objet (aimé)»? L'« Être»? Choisissons «l'être» (note de l'auteur).

ajouté au milieu du texte original : «À l'instant j'ai été éveillé par les battements de cœur précipités...» La petite scène décrite immédiatement après le rêve entre en résonance avec les premières pages du livre où Montherlant évoquait ses souvenirs de la guerre. Il y était déjà question d'un rêve lié à la mort, du même positionnement douloureux dans un lit de douleurs :

Je m'étais couché sur le côté gauche vers le mur, une de mes jambes croisée sur l'autre, comme les lions mourants. [...] En définitive, j'avais souffert plutôt moins que les autres, et c'était tout ce que je n'avais pas souffert dans la guerre qui remontait dans ces rêves de l'après-guerre, comme si mon lot de souffrance devait être comblé. Il y a des malheureux pour qui, vivre, c'est leur sommeil : ils s'y oublient. Pour moi, le sommeil était le mal-vivre. Je ne me couchais qu'avec malaise et appréhension. (12)

Entre le début et la fin du livre, Montherlant crée un dialogue dominé par la souffrance, renouant ainsi avec cette forme de généralisation qui caractérise l'écriture de moraliste. À ce titre, la phrase finale, ajoutée dans la deuxième version, et empruntée à Quo vadis, étend l'expérience individuelle en la replaçant dans l'histoire et dans la culture : «Les prétoriens cernaient les bosquets sur les berges de l'étang d'Agrippa. » La citation, peut-être un peu pompeuse, offre le mérite de relier le moment de l'écriture au moment de l'enfance (des lectures d'enfance), d'inscrire l'aventure humaine de Montherlant dans une généralité qui la dépasse sans la sublimer et enfin de constituer une sorte de refrain qui scande la totalité du chapitre. Intégrer le premier texte à une structure plus grande, créer une chambre d'échos pour assurer la cohérence du livre et le dynamisme de la pensée, telle est sans doute le défi que doit relever l'écrivain qui réécrit. Mais le travail ne se limite pas à un effort de soudure et d'orchestration. «Je recopie ici la note que j'écrivis le matin du 12 décembre» : depuis 2003, le lecteur sait que cette affirmation est mensongère, que Montherlant ne se contente pas de recopier, mais que les modifications qu'il apporte à son premier texte en infléchissent nettement la portée et la réception.

Le phénomène de réduction créatrice ne s'impose guère dans ces pages à quelques exceptions près peut-être (élimination de la répétition du verbe «empoisonner», par exemple, disparition de toutes les informations inutiles : l'heure du réveil, la présence d'un comparse). La plupart du temps, c'est même à une légère expansion ${ }^{10}$ que procède la réécriture : «j'en avais quand même une certaine illusion» devient «Il m'est arrivé quand même d'en avoir une certaine illusion»; «Qui pourra comprendre» devient «Dans une société nouvelle qui pourra comprendre»; «d'aujourd'hui» devient «où elle existe aujourd'hui ». Cette fois-ci, plutôt que de jouer sur la densité imitée de la maxime, Montherlant mise sur une forme d'amplification qui, d'une version à l'autre, tend à donner à ce rêve matinal la forme d'un véritable «tombeau », dans tous les sens, funéraire et littéraire, du terme.

Du brouillon au texte publié, Montherlant donne une très forte dimension religieuse à la scène qu'il raconte à son lecteur. On ne reviendra pas sur la formation catholique du jeune élève de Sainte-Croix de Neuilly, sur son athéisme, voire son paganisme, constamment revendiqués, ni sur la tendresse qu'il conserve pour cet univers culturel qui 
inspire tant de ses textes. Retrouvant le réflexe de nombreux écrivains qui cherchent à sacraliser un événement ou un personnage, à grandir une situation, il emprunte presque spontanément un vocabulaire religieux, quitte à lui imprimer une marque personnelle. Ainsi, la seconde version ajoute à la première les termes de «visage béni » (la «sainte face» n'est pas loin), d' «apparition» ou encore de «visitation», ce dernier terme selon un usage assez particulier. Que faut-il entendre par «visitation»? On a le sentiment que Montherlant associe mentalement et dans le même mot deux scènes célèbres du Nouveau Testament : à proprement parler, la «Visitation» désigne cette visite, très souvent représentée par les peintres, que sainte Élisabeth enceinte de Jean-Baptiste rend à Marie, elle-même enceinte du Christ. Mais inscrite en filigrane dans cette première référence, comment ne pas penser encore à une autre visite, celle de l'archange Gabriel, venu annoncer à la Vierge qu'elle porte en elle le Sauveur? C'est également une «Annonciation» qui est présente en creux dans ce texte écrit quelques jours avant Noël. Et dans ce dépliement des références religieuses imbriquées les unes et les autres comme des poupées gigognes, le lecteur, par la puissance du rêve, assiste à une véritable «Résurrection», qui a la consistance des nuages et qui durera ce que durent les rêves.

Tous les moyens de la réécriture sont mis en œuvre pour donner à cette apparition - on n'est pas loin non plus du flaubertien «Ce fut comme une apparition »-son plus haut degré d'intensité et sa force de surgissement. Montherlant ménage les contrastes en mettant en place un fond de banalité sur lequel l'événement et le «visage béni » se détacheront d'autant plus fortement. En ajoutant «Curieusement, je ne rêve pas », qui confronte le sujet à une expérience commune, en glosant à propos du «rêve très court», «sans doute très court, pas plus qu'une image», il réduit volontairement l'assurance du sujet éveillé, l'épaisseur de l'événement, et prépare la violence de l'affect et du retentissement. Il n'est pas jusqu'à l'humour ajouté lors de la réécriture qui ne soit chargé de dire l'intensité de la présence («la présence réelle» ?) par cette forme de distance qu'on appelle la pudeur ${ }^{11}$.

Même si les nuages l'emportent toujours, la sacralisation du rêve, le désir propre à la réécriture de construire un tombeau créent dans Aimons-nous... une espérance que l'on ne trouvait pas dans le brouillon. En coupant la fin de la version originale, Montherlant réduit considérablement le nihilisme et la mélancolie, osant même résister à la vanité des choses. À l'inconsistance du rêve et des nuages, il oppose la durée d'un tombeau qui à défaut de l'éternité a un peu de temps devant lui. La version d'Aimons-nous... manifeste ainsi une beaucoup plus grande confiance : dans les talents de l'écrivain qui ne doute plus de sa capacité à recréer l'événement («même si je savais l'exprimer mieux » disparaît); confiance également dans l'ouverture sur le temps que permet la littérature. Seul Proust pouvait croire que la mort de Dieu se compenserait par la transcendance de l'œuvre d'art. Pour Montherlant comme pour la plupart de ses contemporains, la littérature se contente de fixer un temps les nuages avant que le ciel ne reprenne son perpétuel mouvement de métamorphose. Quand la première version décrit l'effacement progressif du rêve appelé à se dissiper et avec lui la vérité qu'il impose au dormeur, la version publiée construit une sorte de monument au premier grand amour.

Mais l'on n'est pas quitte avec ce texte - et avec la réécriture - quand on a rendu hommage à l'émotion qui s'en dégage. En effet, la lecture attentive des deux versions et leur comparaison font apparaître une autre dimension, une forme de ruse supérieure, qui, dans un premier temps, semble contredire la vérité de l'affect. «Je recopie simplement... » : le lecteur sait bien que c'est faux et que la simple copie tourne à la réécriture minutieuse. Faut-il accuser l'auteur de mensonge ? On s'en gardera bien. S'il porte un masque, Montherlant ne manque pas de le désigner du doigt, attitude commune à tous les dissimulateurs qui tiennent leur secret à portée de déchiffrement. Quelle est l'identité de ce premier amour qui est tout l'amour? Le lecteur familier de Montherlant reconnaît sans mal le jeune Philippe dont la présence hante 1'auteur depuis L'Exil jusqu'à La Ville dont le prince est un enfant et Les Garçons. Au lieu de le nommer, y compris dans la première version écrite loin du

10. La substitution de «franc cœur» à «bon cœur» évite, sans doute, la banalité du lieu commun. Quand il le faut, Montherlant maintient la répétition d'un mot quand cette répétition lui paraît nécessaire; ainsi les deux versions reproduisent la même redondance : «bouleversant(e) parce qu'il bouleverse».

11. Sur ce fond presque incolore, l'apparition est mise en scène grâce, en particulier, à quelques substitutions de mots : «tirer» à la place de «faire remonter», « découvrir » à la place de «montrer», pour insister sur l'arrachement et la révélation. 
lecteur, Montherlant préfère jouer avec les subtilités de la grammaire française. Si les mots «personne» et «être» peuvent désigner tous les deux indifféremment un homme ou une femme, l'oscillation entre les genres grammaticaux est constante, entretenant une incertitude voulue sur le sexe biologique du «visage béni ». Une note dans la première version mettait ostensiblement l'accent sur la difficulté de nommer l'apparition ( «quelqu'un»? «la personne»? «la créature »?....). Rien de tel dans la seconde version, plus prudente et plus rusée, où la présence du masculin s'estompe plus encore sans pour autant disparaître. Mais l'incertitude générique et sexuelle demeure jusqu'au bout; et c'est cette incertitude qui constitue à la fois le masque et le doigt.

Il appartient au lecteur de savoir lire, de répondre avec vigilance aux signes qui lui sont communiqués dans ce jeu qui travaille sur de l'émotion vive. On s'arrêtera à un seul petit exemple, presque dérisoire par le peu de place qu'il occupe. Quel est le rôle, dans Aimons-nous..., des trois points entre parenthèses [...] qui précèdent le paragraphe commençant par «L'incommunicabilité d'être à être a existé...»? Très régulièrement, le signe typographique désigne une coupure; cette coupure qui déchire un peu le tissu des nuages indique clairement au lecteur qu' on ne lui dit pas tout, qu'on lui cache (par pudeur? par précaution?) quelque vérité bonne à dissimuler. Appel au déchiffrement, invitation au voyage, la coupure ouvre les portes de la lecture à défaut des «portes éternelles » qui ont plutôt tendance à se fermer. Grâce à la publication de Garder tout..., on est maintenant à même de rétablir la pièce manquante du puzzle. Mais la découverte ne vient-elle pas décevoir notre attente ? Ce que Montherlant a coupé et désigné comme coupure, c'est un moment de doute, presque de blasphème ou de déréliction éliminé de la version publiée («J'écris ceci une heure à peine après mon réveil. J'ai voulu noter tout de suite l'impression que m'avait faite ce rêve. Mais déjà le dessin de celui-ci s'efface. Puisse-t-il s'effacer tout à fait. ») La porte n'ouvrait pas sur le secret sexuel du texte ; mais en même temps, ménageant ses pièges sur le chemin de l'errance, le signe nous a bien fait signe. La ruse qui sait si bien ruser avec elle-même a bel et bien appelé le lecteur à ne pas se montrer dupe des mots, à lire entre les lignes, au cœur du palimpseste.

Le discours critique de Montherlant s'accompagne souvent d'affirmations ou de revendications un peu hâtives comme la «sincérité », «la vérité de la vie», le «cri», que l'on n'a pas envie de contester, mais auxquelles on peine à donner une définition claire. Qu'est-ce que la «sincérité »? Quand sommes-nous « sincères »? La « sincérité » peut-elle être la cause efficace et nécessaire de la création ? On a envie de se tourner vers Gide, vers la mauvaise foi sartrienne, vers un moraliste sans illusion comme La Rochefoucauld, pour montrer combien la «sincérité » appartient elle aussi au monde des illusions nuageuses de la reine Jeanne. Mais comme souvent chez Montherlant, l'œuvre fictionnelle ou narrative est plus intelligente que les idées de l'auteur. Quand l'auteur ne cesse de revendiquer une sincérité inaugurale, son œuvre, et tout particulièrement le récit du rêve de décembre 1971, montre à l'évidence que la sincérité relève de l'effet. Il ne s'agit pas de douter de la sincérité du rêveur au petit matin; il s'agit de comprendre que cette émotion de départ ne se dira qu'en acceptant de suivre de longs et difficiles détours. Que nous apprend en effet le travail de l'écriture et de la réécriture? Qu'il est possible de nouer dans la même page l'émotion et la ruse, l'affect et sa maîtrise, l'abandon et le contrôle de soi. Et que ce beau paradoxe est tout simplement la littérature. 
Professeur de littérature française et francophone à l'université CY Cergy Paris, CLAUdE Coste consacre une grande partie de sa recherche à l'œuvre de Roland Barthes (Roland Barthes ou l'art du détour, Hermann, 2016) dont il a édité plusieurs séminaires au Seuil (Comment vivre ensemble, Le Discours amoureux, Sarrasine de Balzac); il codirige l'équipe Barthes à l'ITEM-CNRS. Sa recherche porte également sur les relations de la littérature et de la musique (Orphée ou les sirènes, 2014). En 2010, il coorganise le colloque Lire Montherlant dont les actes ont paru chez Honoré Champion en 2015.

coste.claude@wanadoo.fr

Résumés

\section{Quelques remarques sur la réécriture chez Montherlant : Mais aimons-nous ceux que nous aimons? et Gardons tout en composant tout}

Dans Aimons-nous ceux que nous aimons?, Montherlant revient sur deux œuvres de jeunesse qu'il réécrit partiellement; il propose également un récit de rêve dont une première version parait de façon posthume dans Garder tout en composant tout. Que signifie cette pratique récurrente de la réécriture? Dans toute son œuvre, Montherlant cherche à rester maître de son image, conciliant la vérité de l'affect et les ruses de l'art.

In Mais aimons-nous ceux que nous aimons?, Montherlant returns to two early works to partially rewrite them. The book also offers a dream story, whose first version appears posthumously in Garder tout en composant tout. What does this recurring practice of rewriting mean? Throughout his writing career, Montherlant seeks to remain master of his persona, reconciling the truth of affect with the cunning of art.

In Aimons-nous ceux que nous aimons? kehrt Montherlant zu zwei frühen Werken zurück, die er teilweise umgeschrieben hat; außerdem schlägt er eine Traumerzählung vor, deren erste Version posthum in Garder tout en composant tout erscheint. Was bedeutet diese wiederkehrende Praxis des Umschreibens? In all seinen Werken versucht Montherlant, Herr seines eigenen Bildes zu bleiben, indem er die Wahrheit des Affekts und die Tricks der Kunst in Einklang bringt.
En Aimons-nous ceux que nous aimons?, Montherlant retoma dos obras juveniles y las reescribe parcialmente; al mismo tiempo ofrece el relato de un sueño cuya primera versión aparece, de manera póstuma, en Garder tout en composant tout. ¿Qué significa esta práctica recurrente de la reescritura? En toda su obra, Montherlant trata de controlar permanentemente su imagen, conciliando la verdad de los afectos con las astucias del arte.

Em Aimons-nous ceux que nous aimons?, Montherlant regressa a duas obras de juventude que parcialmente reescreve; propõe também uma narrativa de sonho, cuja primeira versão aparece postumamente em Garder tout en composant tout. O que significa esta prática recorrente da reescrita? Em toda a sua obra, Montherlant procura manter-se fiel à sua imagem, conciliando a verdade do afeto e as artimanhas da arte.

In Mais aimons-nous ceux que nous aimons ?, Montherlant ritorna su due opere giovanili che riscrive parzialmente, proponendo anche il racconto di un sogno, una prima versione del quale appare postuma in Garder tout en composant tout. Cosa significa questa pratica ricorrente della riscrittura? In tutta la sua opera Montherlant cerca di restare padrone della propria immagine, conciliando la verità dell'affetto e le astuzie dell'arte. 